\title{
Truth Table Tasks: Irrelevance and Cognitive Ability
}

Aline Sevenants, Kristien Dieussaert and Walter Schaeken

University of Leuven

Key words: conditionals, truth table tasks, cognitive ability, mental model accounts, suppositional theory

\section{Corresponding author:}

Aline Sevenants

\section{Aline.Sevenants@psy.kuleuven.be}

University of Leuven, Laboratory of Experimental Psychology

102 Tiensestraat, B-3000 Leuven, Belgium

Tel: $+32(0) 16 / 32.59 .52$

Fax: $+32(0) 16 / 32.60 .99$ 


\begin{abstract}
Two types of truth table task are used to examine people's mental representation of conditionals. In two within-subjects experiments, participants either receive the same tasktype twice (Experiment 1) or are presented successively with both a possibilities task and a truth task (Experiment 2). In Experiment 3 it is examined how people interpret the threeoption possibilities task and whether they have a clear understanding of it. The present study aims to examine for both task-types how participants' cognitive ability relates to the classification of the truth table cases as irrelevant, and their consistency doing so. Looking at the answer patterns, participants' cognitive ability influences their classification of the truth table cases: a positive correlation exists between cognitive ability and the number of falseantecedent cases classified as 'irrelevant', both in the possibilities task and the truth task. This is in favor of a suppositional representation of conditionals.
\end{abstract}

Key words: conditionals, truth table task, cognitive ability, mental model accounts, suppositional theory 


\section{Acknowledgments}

This research was carried out with the financial support of the National Council for Scientific Research - Flanders, Belgium (FWO grant G.0320.05) and the Odysseus Type 1 project granted to Prof. Dr. I. Douven. We want to render thanks to David Over, Pierre Barrouillet and an anonymous reviewer for their helpful comments on this manuscript. We are also grateful to Jean-François Bonnefon and Klaus Oberauer for their inspiring suggestions. 


\section{Truth Table Tasks: Irrelevance and Cognitive Ability}

People's understanding and mental representation of conditionals have been the subject of a wealth of studies. Conditionals are sentences with the following grammatical construction: 'If A then $\mathrm{C}^{\prime}$, with $\mathrm{A}$ being the antecedent and $\mathrm{C}$ the consequent of the clause. E.g.: If the letter is $a T$, then the number is a 5. Combining the truth-value of the antecedent and the consequent, four cases arise:

1. True antecedent - True consequent (TT): $a T$ and $a 5$

2. True antecedent - False consequent (TF): a T and not a 5

3. False antecedent - True consequent (FT): not $a$ T and $a 5$

4. False antecedent - False consequent (FF): not a $T$ and not a 5

The TT case and the TF case ( 1 and 2) are referred to as the true-antecedent cases and the FT and FF cases ( 3 and 4 ) as the false-antecedent cases.

There has been substantial debate in reasoning literature concerning the processes and representations underlying people's understanding of conditional assertions. Two main theories accounting for the mental representation of conditionals are the Mental Models Theory (Johnson-Laird \& Byrne, 1991; 2002; Johnson-Laird, Byrne, \& Schaeken, 1992) and the Suppositional Theory (Evans \& Over, 2004; Evans, Handley, Neilens, \& Over, 2007; 2008; Evans, Over \& Handley, 2003a). The starting point for much of the debate between these theories has been the truth table task, since both theories claim support for their account, each from one version of the task but not from the other: the mental models theory from the possibilities task and the suppositional theory from the truth task. In the classical possibilities task, people have to indicate for each of the four possible antecedent-consequent cases whether that specific combination is either possible or impossible with respect to the given rule. In the truth task, participants are asked to evaluate for each of the four cases whether the 
combination makes the given rule true, false or is irrelevant. The suppositional theory and the mental models theory make different predictions about the mental representation of conditionals. According to the mental models theory, people reason with representations resembling truth tables stemming from the two-valued standard logic (also referred to as 'logical' answer patterns in reasoning literature), judging the TT case, the FT case and the FF case to be possible according to the rule in a possibilities task. The only case that falsifies the conditional is TF. According to the suppositional theory, people reason with representations matching three-valued truth tables, including 'irrelevant' as a third option next to 'true' and 'false'.

A wealth of research has indeed questioned whether classical two-valued logic is to be considered as an adequate normative system for conditional reasoning. Wason $(1966,1969)$ was the first to introduce in psychological literature the notion of 'defective truth tables', in which false antecedent cases (FT and FF) are considered to be irrelevant rather than true. In the defective truth table view, people judge that false-antecedent cases have nothing to do with the truth or falsity of a conditional and are therefore labelled as 'irrelevant'. This is referred to by Evans and Over (2004) as a 'truth value gap' for the false-antecedent cases. De Finetti $(1967,2008)$ also departs from standard two-valued logic introducing a third value for the false antecedent cases in conditional events: "null". This truth table, with the false antecedent cases being void, is the so-called 'de Finetti Table' and is comparable to the defective truth table described above (see also Politzer, Over \& Baratgin, 2010). In the remainder of this paper the terminology two-valued vs. three-valued will be used.

The suppositional theory (Evans \& Over, 2004; Evans et al., 2007; 2008; Evans et al., 2003a) provides a detailed account of the three-valued truth tables yielded by the truth task: People evaluate conditionals by means of the Ramsey test. That is, they "hypothetically add $p$ to their stock of knowledge and evaluate their degree of belief in q given p" (Ramsey, 
1931/1990 p.247). So they first estimate the probability of the consequent and the antecedent occurring together (the TT case), and then estimate the probability of the antecedent together with the non-occurrence of the consequent (the TF case). The combination of these two stages then leads to an estimation of the probability of occurrence of the consequent, given the antecedent: $\mathrm{P}(\mathrm{C} \mid \mathrm{A})$. Running this Ramsey test, participants disregard the false-antecedent cases and only focus on those cases in which the antecedent is true, resulting in a three-valued truth table pattern with a truth value gap for the false antecedent cases. For the classical possibilities task, a 'possible' judgment is predicted however since the suppositional theory assumes that false antecedent cases are irrelevant for evaluating a conditional, but that these cases are possible given the conditional. There are no predictions from the suppositional theory about the irrelevance judgments in a three-option possibilities task to be found in reasoning literature, since the classical possibilities task does not allow for three-valued answer patterns. However, false-antecedent 'not-p' cases are consistent with 'if $p$ then $q$, even when this is a suppositional or probability conditional. In the probabilistic approach this means that $\mathrm{P}($ not-p) and $\mathrm{P}($ if $\mathrm{p}$ then $\mathrm{q})$ can both be high and that $\mathrm{P}($ not- $\mathrm{p})$ can be high when $\mathrm{P}($ if $\mathrm{p}$ then $\mathrm{q})$ is high.

Evans et al. (2007, 2008) show that the participants who are responding according to three-valued patterns in a truth task, judging the false antecedent cases to be irrelevant, are those with the highest score on an intelligence test (AH4-Group test of General intelligence, Heim, 1970): These are the ones that are able to complete both stages of the Ramsey test and thus to consider both $\mathrm{A} C$ and $\mathrm{A} \neg \mathrm{C}$. The ones not able to do so consider only the first stage, i.e. the TT case or the probability of the consequent and the antecedent occurring together. These participants do not have enough cognitive resources to also estimate the probability of the antecedent together with the non-occurrence of the consequent (the TF case) and give a conjunctive answer pattern (TFFF). 
The mental models theory provides an account of the two-valued answer patterns yielded by the possibilities task (Barrouillet \& Lecas, 1998; Johnson-Laird, 2006; JohnsonLaird et al., 1991, 2002; Johnson-Laird et al., 1992). According to the mental models theory, when people have to judge whether a situation is possible given a conditional rule, as is the case in the possibilities task, they list all possibilities compatible with the conditional rule. In line with the 'truth principle', reasoners construct mental models of the possibilities compatible with the premises, but they initially and by default do not represent what is false. Therefore, their conclusion is based on the initial model: $[\mathrm{A}] \mathrm{C} \ldots$

Under certain circumstances people make 'mental footnotes' about the falsity of clauses (represented by the ellipsis). If they are able to retain these footnotes, reasoners can flesh out the implicitly represented information into fully explicit models, representing clauses even when they have false antecedents:
A C
$\neg \mathrm{A} \quad \mathrm{C}$
$\neg \mathrm{A} \neg \mathrm{C}$

These three possibilities correspond to the rows of the truth table in which the material implication is true, including the false-antecedent cases FT and FF.

According to the mental models theory (Johnson-Laird, 2006; Johnson-Laird \& Byrne, 2002), the people with the highest working memory capacity are able to look beyond the initial model (A C) and consider all possibilities compatible with the conditional, including the false-antecedent cases, leading to two-valued answer patterns (TFTT). The people with lower working memory capacity however do not process beyond the initial model and respond according to a conjunctive (TFFF) or a three-valued pattern, judging the false antecedent cases as irrelevant.

A revised mental models account for the mental representation of conditionals comes from Barrouillet, Gauffroy and Lecas (2008a). They make the distinction between two different processes relying on the same mental model representation: reasoning about 
possibilities given the truth of assertions (as in the possibilities task), and reasoning about the truth of assertions given possibilities (as in the truth task). According to them, the mental models theory can account for both kinds of reasoning and thus for the results of both kinds of tasks trough an extension of 'the truth principle'. This principle, meaning that people only construct mental models of the possibilities compatible with the premises, states that "each mental model of a set of assertions represents a possibility given the truth of the assertions, and each mental model represents a clause in these assertions only when it is true in that possibility" (Johnson-Laird \& Byrne, 2005, p. 653). Barrouillet et al. (2008a) suggest adding to this principle of truth: "The possibilities that make the assertions true give rise to explicit mental models whereas the other lead to models that remain initially implicit" (p. 16). This modification leads on the one hand to the prediction that false antecedent cases will be judged as possible in the possibilities tasks since they are compatible with the true conditional. These false antecedent cases are judged to be irrelevant with respect to the truth of the conditional in the truth task on the other hand, since these models remain implicit. False antecedent cases do not make the conditional true, but the conditional cannot be false in these cases either because they can occur given the conditional, leading to an irrelevance judgment for the false antecedent cases and thus to three-valued answer patterns in the truth task.

In the present study, we will administer both the truth task an extended three-option possibilities task in which 'irrelevant' is added as an answer option next to 'possible' and 'impossible' of the classical possibilities task, in order to be able to replicate Sevenants, Schroyens, Dieussaert, Schaeken and d'Ydewalle (2008). Using the three-option possibilities task is the only way an explicit comparison between the results yielded by both types of truth table tasks and the reasoning processes underlying them is enabled, since the addition of 'irrelevant' as a third answer alternative to the possibilities task allows for irrelevance judgments in both tasks, and not only in the truth task. Sevenants et al. (2008) found that a 
substantial group of participants classified false antecedent cases as irrelevant with respect to the conditional rule, both in the classical truth task and in the possibilities task with 'irrelevant' as a third option. The finding that a substantial group of participants gives a threevalued answer pattern on the possibilities task is at odds with the mental models account of Johnson Laird and Byrne (2005), and with the modified mental models theory of Barrouillet et al. (2008a), both predicting two-valued answer patterns for the possibilities task. This is the reason why we want to replicate the results of Sevenants et al. (2008), using both the truth task and the three-option possibilities task.

From previous studies we can conclude that task characteristics have a major impact on the response patterns on truth table tasks (Dugan \& Revlin, 1990; Thompson, 2000). Not only the wording (possibility vs. truth) and the number of answer options (two vs. three), but also task directionality (rule-to-situation vs. situation-to-rule) and negation type (implicit vs. explicit) influence participants' answer patterns (Sevenants et al., 2008). Task characteristics however are not the sole determinant of the classifications yielded by the truth table task. Individual differences are another important determinant, and more specifically cognitive ability. There is some debate in reasoning literature concerning the relation between cognitive capacity and the mental representation of conditionals. Shedding light on this debate is the second aim of the present study. The three-valued answer patterns are a key factor since the mental models theory and the suppositional theory have opposite predictions about it: According to the mental models theory, people with a low working memory capacity are not able to process further than the initial model, giving a conjunctive or three-valued answer pattern. According to the suppositional theory, the participants with the highest intelligence are the ones who are able to complete the two stages of the Ramsey test, resulting in a threevalued answer pattern (Evans et al., 2007, 2008). Allowing for three-valued patterns to occur in both task-types, in the present experiments we focus on the relation between cognitive 
ability and the nature of the answer patterns in order to shed light on the opposite predictions of the mental models theory and the suppositional theory regarding the relation between cognitive ability and the nature of the response patterns on the truth table task.

Finally, with the design of the present experiments, we want to check participants' consistency across and between the two truth table task-types. Two within-subjects experiments are conducted in which the participants either receive the same task twice (Experiment 1) or are presented successively with both a three-option possibilities task and a truth task (Experiment 2). More specifically, we want to examine how consistent participants are in their classification of the truth table task cases and if they are not, in what direction they shift. This is important because we want to be sure that participants really carry out the task as we expect them to, instead of giving a random response as might be the case in a computerized tasks.

In sum, the aim of the present study is twofold: Firstly, focusing on task characteristics, we want to examine how participants classify false antecedent cases across and between the two truth table task-types, given the debatable status of 'irrelevant' in the possibilities task. So two within-subjects experiments are conducted in which the participants either receive the same task twice (Experiment 1) or are presented successively with both a three-option possibilities task and a truth task (Experiment 2). Secondly, next to the influence of task characteristics of the truth table task, we are interested in the role of cognitive ability. It is aimed to investigate for both task-types whether the nature of participants' judgments of the false antecedent cases is related to their cognitive ability.

\section{Experiment 1}

In a previous study, Sevenants et al. (2008) observed more two-valued answer patterns in the possibilities tasks and more three-valued answer patterns in the truth tasks, but still there was 
a considerable amount of three-valued patterns yielded by the possibilities tasks and some two-valued patterns yielded by the truth tasks. It was concluded that irrespective of the tasktype, some participants seem to have a two-valued representation of conditionals whereas others have a three-valued representation. Oberauer, Geiger, Fisher, and Weidenfeld (2007) pointed out however that only when participants produce the same response pattern repeatedly within the same task-type, the results can attributed to their interpretation of the conditional rather than to random fluctuations. The first aim of the present experiment therefore is to examine whether we can replicate the observed difference between both types of truth table tasks when the same type of truth table task is administered two times, especially regarding the irrelevance judgments of the false antecedent cases. Presenting the participants twice instead of once with the same truth table task minimizes the odds that the observed answer patterns are just due to random fluctuations. Secondly, next to a replication of previous results, we aim to examine how participants' answer patterns relate to their cognitive ability unlike other experiments in literature allowing for three-value answer pattern to occur in both task-types. This relation with cognitive ability is an issue that remained unanswered by Sevenants et al. (2008). Who are the participants responding with three-valued answer patterns?

\section{Method}

\section{Participants}

Participants were 91 first-year Psychology students at the University of Leuven (17-25 years of age, $M=18.4$ ) taking part in this experiment in partial fulfillment of course requirements. All of them were logic-laymen. 


\section{Materials and Procedure}

In reasoning literature there is a difference in how the concept of cognitive ability is exactly made concrete. According to the mental models theory (Johnson-Laird \& Byrne, 2002), cognitive ability is defined in terms of working memory capacity or the ability to have several possibilities in mind at the same time (Johnson Laird, 2006). In the suppositional theory, cognitive ability is more in terms of general intelligence (Evans et al, 2007, 2008). Since this difference in how the concept of 'intelligence' is made concrete might have an influence on how individual differences relate to the performance on the truth table task, we will administer two tests of cognitive ability in the present study: a working memory capacity test as well as a more general intelligence test.

\section{Working Memory Measure (Gospan)}

Working memory capacity was measured during a group session prior to the truth table task experiments using a version of the Operation Span Task (Ospan; La Pointe \& Engle, 1990) adapted for group testing (De Neys, d'Ydewalle, Schaeken, \& Vos, 2002). The Ospan is a classic working memory measure that primarily reflecting central executive capacity (Engle, Tuholski, Laughlin, \& Conway, 1999) in which participants solve series of simple mathematical operations while attempting to remember a list of unrelated words. They were presented with a series of simple mathematical operations on the computer screen which they had to solve while attempting to remember a list of unrelated words. First, an operation was presented on the computer screen (e.g., IS (4/2) - $1=5$ ?). After reading the operation silently, the participants pressed a key to indicate whether the answer is correct or not. Responses and response latencies were recorded. After the participant had given his response, a word (e.g., 'ball') was presented for $800 \mathrm{~ms}$. After a number (ranging from 2 to 6) of operation-word pairs had been presented, participants were asked to write down in the correct order the word they had been presented. This means that participants had to solve the operations while 
attempting to remember the presented words. In the Gospan, 15 sets of operation-word pairs were tested (three sets of each length, ranging from two to six operation-word pairs). The Gospan score (maximum 60) is the sum of the recalled words for all sets recalled completely and in correct order.

\section{Cognitive Reflection Test (CRT)}

The second test of cognitive ability we administered is the Cognitive Reflection Test (CRT; Frederick, 2005). We have three reasons for choosing this test: First of all, it only consists of three questions and is easily administrable for large groups of students. Secondly, more than the Gospan it is a measure of general intelligence (Navarrete, De Neys, \& Santamaria, 2008) correlating .44 with the Scholastic Achievement Test (SAT), .46 with the American College Test (ACT) and .43 with the Wonderlic Personnel Test (WPT) in a population of American university students (Frederick, 2005), and .35 with a short version of the Raven Progressive Matrices (Sevenants, Dieussaert, Schaeken, \& Barrouillet, unpublished manuscript). Finally, the CRT is conceived as a measure for reasoning beyond the quick and automatic heuristic response (Frederick, 2005). Since reasoning beyond this first quick and automatic response is both according to the suppositional theory and the mental models theory what people with high cognitive ability do, the CRT seems an appropriate measure of cognitive ability.

The CRT (Frederick, 2005) was administered during the same session as the truth table tasks. All participants received a sheet on which the instruction 'Below are several problems that vary in difficulty. Try to answer as many as you can.' appeared, followed by the three items of the CRT. The items as well as the instructions were presented in Dutch. The CRT score is the sum of the number of correct items, ranging from 0 to 3 .

(1) A bat and a ball cost $\$ 1.10$ in total. The bat costs a dollar more than the ball. How much does the ball cost? cents 
(2) If it takes 5 machines 5 minutes to make 5 widgets, how long would it take 100 machines to make 100 widgets?

minutes

(3) In a lake, there is a patch of lily pads. Every day, the patch doubles in size. If it takes 48 days for the patch to cover the entire lake, how long would it take for the patch to cover half of the lake? days

\section{Truth Table Tasks}

After completing the CRT, participants were run in two groups with task-type as a betweensubjects variable. One group $(n=47)$ performed the three-option possibilities task twice, while the other group $(n=44)$ was presented with the truth task twice. Both groups received the same instructions, appearing on the computer screen and explaining that the purpose of the experiment was to examine how people reason with conditionals. The instructions also contained the description of a machine producing cards with a letter on the front side and a number on the back, always doing so following a certain rule, for example 'If there is an $A$ on the front, there is a 2 on the back of the card, ${ }^{,}$. Participants could read that in the upcoming task they were going to see the content of eight specific cards produced by that machine, and that per card they had to evaluate the compatibility of the card with the card-producing rule. Participants were then provided with a concrete example of a conditional rule, as well as with an example of the item in the actual task (no correct answers were provided). The instructions were followed by one practice trial. In the instructions it was made clear to the participants that they had to judge each time, for one specific card taken randomly from the pack, whether it was possible/impossible or true/false that this specific card was produced by that machine.

\footnotetext{
${ }^{1}$ Note that the participants are asked to judge the cards based on a rule, e.g., 'If there is an A on the front, there is a 2 on the back of the card'. They have to assume that the machine produces the cards following this rule, but it is never explicitly mentioned to the participants however that they have to consider this rule to be 'true'. The conditional rule is thus presented as 'given that if $\mathrm{p}$ then q' and not as 'given that if $\mathrm{p}$ then $\mathrm{q}$ is true'. This is an important difference, hinging on the distinction between the pleonastic use of 'true' as is the case in the present study, and its use to refer to an objective state of affairs (see also Adams, 1998; Douven \& Verbrugge, 2010; Edgington, 2003; Politzer et al., 2010).
} 
This because the scope of the conditional rule cannot be a whole pack of cards (as is the case for general conditionals, which are not made definitely true by only one "p \& q" case, see also Schroyens, 2010 on the induction problem) but has to apply only to a single card taken randomly from the pack (Evans et al., 2003, p. 333). The conditional rule in the present truth table tasks is singular and has a definite description, e.g., "If the letter is a T..." and does not begin with an indefinite quantifier characterizing general conditionals, as in "If a card had a $\mathrm{T}$ on the front ..." or "If any card has a $\mathrm{T}$ on the front ...".

In both task-type conditions, all participants were successively presented twice with four items. Each item consisted of the abstract conditional rule followed by one of the four combinations of occurrence and non-occurrence of A and C. Only explicit negations were used. Concretely, all participants were presented twice with each of the following situations. For each of both runs of the task other letters and numbers were used.

- $\quad$ Rule: if the letter is a $T$, then the number is a 5 Situation: 'a $T$ ' and 'a 5 '

- $\quad$ Rule: if the letter is a T, then the number is a 5 Situation: ' $a$ T' and 'not a 5'

- $\quad$ Rule: if the letter is a $T$, then the number is a 5 Situation: 'not a $T$ ' and 'a 5 '

- $\quad$ Rule: if the letter is a $T$, then the number is a 5 Situation: 'not a $T$ ' and 'not a 5 '

More concretely, in the possibilities tasks, participants had to evaluate for each of the four situations whether 'the situation is possible according to the rule', 'the situation is impossible according to the rule', or whether 'the situation is irrelevant according to the rule'. In the truth tasks, participants had to evaluate for each of the four antecedent-consequent combinations whether 'the situation makes the rule true', 'the situation makes the rule false', or whether 'the situation is irrelevant with respect to the truth of the rule'. No feedback was provided. For each item, the rule followed by one of the four situations appeared on the 
screen. Each item appeared on a different screen. Presentation order of the items was randomized. In order to keep the possibilities task evenly to the utmost to the truth task, we chose for the evaluation format of both truth table task-types.

Tasks were constructed with 'E-prime' software (Psychological Software Tools, Pittsburgh, PA) and presented to the participants on individual standard PCs in a self-paced manner. Responses were given with the arrow-keys on an AZERTY keyboard. The Truth table Task experiment lasted between 5 and 10 minutes, the CRT less than 5 minutes and the Gospan about 20 minutes. All parts of the experiment were presented in Dutch.

\section{Results and Discussion}

Before we turn to the results of Experiment 1, we will briefly explain in the paragraphs below some indices we calculated in order to analyze the data.

Regarding the judgments on the truth table task, we divided the truth tables into two components: the true-antecedent cases (TT and TF) and the false-antecedent cases (FT and FF). According to both the suppositional theory and the MMT, participants should judge the TT case to make the conditional true and the TF case to falsify the conditional. By analogy with Evans et al. (2007) we combined these judgments into the logic index (L-I): per participant the TT situations judged as 'possible' or 'true' plus the TF cases judged as 'impossible' or 'false', (depending of the type of truth table task they received). Regarding the false-antecedent cases, we computed the false-antecedent index $(F A-I)$, which is the sum per participant of false-antecedent cases (FT and FF) judged to be 'irrelevant'. Both the L-I and the FA-I range from 0 to 4.

In addition to the logic index and the false-antecedent index as used by Evans et al. (2007, 2008), we computed three more indexes. According to the suppositional theory, participants should judge the TT case as 'possible' or 'true', the TF case as 'impossible' or 'false' and both the FT and the FF case as 'irrelevant', especially in the truth task (there are no 
predictions about the judgments of the false antecedent cases in the possibilities task since the classical possibilities task classically provides no irrelevance option). We combined these judgments into the suppositional theory index (ST-I), ranging from 0 to 8 per participant. Moreover, we calculated the material implication index (MI-I), summing per participant the number of TT cases judged as 'possible' or 'true', TF cases as 'impossible' of 'false', and FT and FF cases as 'possible' or 'true' (range 0-8). This is the classification pattern the mental models theory would predict for those participants with [A] $\mathrm{C}$ as initial model who are able to flesh out into fully explicit models. Finally we computed a conjunctivity index (Conj-I) with a range from 0 to 8 . This classification pattern in which only the TT case is judged as 'possible' or 'true' and the three other cases as 'impossible' or 'false' is predicted both by the mental models theory and the suppositional theory for the participants with the least cognitive resources, unable to reason beyond the initial model or to engage in the second stage of the Ramsey Test.

First of all, regarding the difference between the possibilities task and the truth task, we replicated the finding that truth tasks yield more irrelevance answers than do possibilities tasks (Sevenants et al., 2008): The FA-I was higher for the truth tasks $(M=2.18, S D=1.45)$ than for the possibilities tasks $(M=1.21, S D=1.23, t(89)=3.44, p<.001)$. In addition, more 'possible' judgments of the false-antecedent cases were yielded by the possibilities task than 'true' judgments of the false-antecedent cases were yielded by the truth task: The MI-I is higher for the possibilities tasks $(M=6.02, S D=1.45)$ than for the truth tasks $(M=4.48, S D$ $=1.07, t(89)=5.75, p<.001)$. This shows that there is a clear difference between the possibilities task and the truth task concerning the judgment of the false-antecedent cases: both task-types are asking a different question, eliciting a different kind of representation of information. Asking participants to make a judgment of truth recruits the type of information that the suppositional theory refers to, whereas asking for a judgment of possibility recruits 
the type of information that the mental models theory refers to (see also Barrouillet et al., 2008; Sevenants et al., 2008).

Although truth tasks yield more irrelevance answers than do possibilities tasks, we do replicate the finding of Sevenants et al. (2008) that there is still a considerable amount of participants giving irrelevance judgments on false antecedent cases in the possibilities task (21\%). This is problematic for the mental models theory of Johnson Laird and Byrne (1991; 2002) and for the modified mental models account of Barrouillet et al. (2008a), predicting possible judgments for the false antecedent cases in the possibilities task.

With respect to the true-antecedent cases, t-tests do not reveal a different Log-I for the possibilities tasks and the truth tasks, meaning that in both tasks participants equally often classify TT cases as 'possible' or 'true' and TF cases as 'impossible' or 'false'. This was to be expected, since both the mental models theory and the suppositional theory predict this answer pattern for the true-antecedent cases. This observation is in line with both the mental models accounts and the suppositional theory.

Regarding participants' consistency when being presented with twice a possibilities task or twice a truth task, we made the following observation. Inspecting participants' answer patterns (see Table A1), and grouping the TFTT and TFFT classification patterns into 'twovalued patterns' and TFII and TFFI into 'three-valued patterns' (see Table A2), we see that $56 \%$ of the participants showed consistency, giving either a two-valued or a three-valued answer pattern on both runs of the same task: $33 \%$ of the participants were consistent giving twice a three-valued answer pattern and $23 \%$ giving twice a two-valued one.

Insert Table 1, 2 and Table 3 about here 
Considering the relation between participants' truth table classifications and cognitive ability, we observed a similar pattern of results for both measures of cognitive ability. Both for the possibilities task and the truth task, there was a significant correlation between the CRT and both the FA-I (see Table 1) and the ST-I. Similarly, we found a positive correlation between the Gospan and the FA-I as well as between the Gospan and the ST-I. Separating the analyses of correlations by task, we observe a similar pattern of correlations for both tasktypes. Both for the possibilities task and the truth task, there was a positive correlation between cognitive ability and both the FA-I and the ST-I. However, in the possibilities task this correlation was only significant with the CRT whereas in the truth task only the correlation between the Gospan and both the FA-I and the ST-I was significant (see Table 2 and Table 3).

These correlations are evidence in support of the suppositional theory, which predicts a positive correlation between cognitive ability and the number of false-antecedent cases classified as 'irrelevant'. This finding is in turn problematic for the mental models theory, which predicts participants low rather than high in cognitive ability to judge false-antecedent cases as 'irrelevant' instead of as 'impossible' or 'false': Participants high in cognitive ability should be able to look beyond the initial model, leading to irrelevance judgments, and consider the three fully explicit models leading to a 'possible' judgment. Moreover, in our data we observed a significant negative correlation between the Gospan and the MI-I in the possibilities task and a negative correlation between the CRT and the MI-I truth task, but the latter trend failed to reach significance. This means that the participants with a high rather than a low working memory span are the ones that tend to judge the false-antecedent cases as 'irrelevant', even in the possibilities task (see Table 2 and Table 3), which is the opposite of the predictions of the mental models theories. The fact that it are the most intelligent participants that choose for 'irrelevant' in both task-types, suggests that participants are not 
only doing so because of for example a lack of understanding of what this answer option means. Sevenants et al. (2008) already showed that, asking for a justification of their judgments, not even one participant mentioned to be confused by the presence of the irrelevance option in the possibilities task. Irrelevant can thus be seen as a meaningful option, both in the possibilities task and the truth task. It is referred to by Evans and Over (2004) as a 'truth value gap' for false antecedent cases, in which people judge that these cases have nothing to do with the truth or falsity of the conditional, since the antecedent does not hold. Of course, the wording 'truth value gap' is somewhat infelicitous for the possibilities task as compared to the truth task, since in the possibilities task there are strictly speaking no truth values but rather possibilities to evaluate. Nevertheless, it seems to be the case that false antecedent cases are judged comparably both in the truth task and in the possibilities task with an irrelevance option.

Summarising the results of this first experiment, we replicate that truth tasks yield more irrelevance answers than do possibilities tasks (Sevenants et al., 2008). When looking at the individuals' answer patterns, we find that $56 \%$ of the participants are consistent within task-type. Regarding the association between the judgments on the truth table tasks and participants' cognitive ability, we observe an overall positive correlation between the number of false-antecedent cases classified as 'irrelevant' and participants' intelligence (see Table 1). Moreover, a negative correlation is revealed between the Gospan scores and the MI-I, for the three-option possibilities task. This provides evidence to the three-valued truth table representation of the suppositional theory in which false-antecedent cases FT and FF are judged to be irrelevant with respect to the rule (Evans \& Over, 2004). 


\section{Experiment 2}

In the first experiment of this study, we investigated task-type as a between-subjects variable: participants received either a possibilities task or a truth task twice. In the present experiment, we aim to study participants' classification of false antecedent cases across instead of within tasks in order to be able to detect any possible shift in the answer patterns going from a threeoption possibilities task to a three-option truth task or the other way around. Moreover, as was the case in Experiment 1 of the present study, we are interested in how the classification of the false antecedent cases relates to participants' cognitive ability.

\section{Method}

\section{Participants}

In the present experiment, 77 first-year Psychology undergraduate students (17-23 years of age, $M=18.2$ ) at the University of Leuven took part in partial fulfillment of course requirements. None of them had ever taken a logic-course. Participants were run in two groups. One group $(n=39)$ first received the three-option version of the possibilities task, followed by the truth task. The other group $(n=38)$ first received the truth task and then the three-option version of the possibilities task.

\section{Materials and Procedure}

Materials and procedure were exactly the same as in Experiment 1, as well as the instructions. In the possibilities task, participants had to evaluate for each of the four situations whether that situation was possible, impossible or irrelevant according to the rule. In the truth task participants had to evaluate for each of the four possible antecedentconsequent combinations whether the combination made the given rule true, false, or was irrelevant with respect to the truth of the rule. The only difference with Experiment 1 was 
that, instead of being presented twice with only one version of the truth table task, in the present experiment participants were presented both with the possibilities task and the truth task, thus with task-type as a within-subjects variable. All participants thus had to judge the four cases of the truth task plus the four cases of the possibilities task, the order of the tasktypes being randomized.

The same cognitive ability measures as in Experiment 1 were used, so prior to the truth table task experiment, participants were presented with both the CRT (Frederick, 2005) and the Gospan working memory capacity measure (De Neys, d'Ydewalle, Schaeken, \& Vos, 2002).

\section{Results and Discussion}

Analyzing the results we computed the same indices as in Experiment 1: A logic index $(L-I$; TT case judged as 'possible' or 'true' plus TF cases classified as 'impossible' or 'false'), a false-antecedent index (FA-I; the sum per participant of false-antecedent cases judged as 'irrelevant'), a suppositional theory index (ST-I; TT cases classified as 'possible' or 'true', TF cases as 'impossible' or 'false' and both false-antecedent cases as 'irrelevant'), a material implication index (MI-I; summing the TT cases judged as 'possible' or 'true', TF cases as 'impossible' of 'false', and FT and FF cases as 'possible' or 'true') and finally a conjunctivity index (Conj-I; summing the TT cases as 'possible' or 'true' and the other three cases as 'impossible' or 'false'). Since participants in the present experiment are presented with one possibilities task and one truth task (opposed to twice a possibilities task OR twice a truth task in Experiment 1) the range of the indices is halved compared to Experiment 1 when tasks are discussed separately.

With respect to the difference between the possibilities task and the truth task, we replicated the finding that on the one hand truth tasks yield more irrelevance answers than do possibilities tasks. However, more than a quarter of the participants still produce a three- 
valued truth table pattern in the possibilities task (Sevenants et al., 2008). As was the case in Experiment 1 of the present study, the FA-I for the truth task $(M=.91, S D=.80)$ was higher than for the possibilities task $(M=.60, S D=.67, t(152)=2.62, p<.001)$. More 'possible' judgments of the false-antecedent cases on the other hand are yielded by possibilities tasks than 'true' judgments for these cases by truth tasks: The MI-I was higher for the possibilities tasks $(M=2.47, S D=.79)$ than for the truth tasks $(M=2.20, S D=.61, t(152)=12.20, p<$ .001). This means that there seems to be a clear difference between the possibilities task and the truth task with respect to the classification of the false-antecedent cases, but not for the true-antecedent cases (t-tests do not reveal a different Log-I for the possibilities tasks and the truth tasks). This was expected since the mental models theory and the suppositional theory have different predictions regarding the false-antecedent cases, but for the true-antecedent cases both theories predict a classification of TT as 'possible'/'true' and of TF as 'impossible' or 'false'.

As we did in the previous experiment, we examined the individual answer patterns (see Table B1) and more specifically the classification of these patterns into two-valued and three-valued ones. Table B2 shows the nature of the answer patterns (two-valued or threevalued) yielded by the succession of one run of a possibilities task and one run of a truth task. $37 \%$ of the participants were consistent, giving either a two-valued or a three-valued answer pattern on both task-types. $21 \%$ of the participants responded according to a three-valued answer pattern twice and $16 \%$ did so according to a two-valued pattern. This means that for more than one third of the participants, their individual reasoning strategy results in consistent answer patterns over both tasks. $18 \%$ of the participants were not consistent but made a shift from two-valued to three-valued or from three-valued to two-valued. It is important to note that all participants shifted into the direction expected from Experiment 1 and from Sevenants et al. (2008): from two-valued to three-valued when they received the possibilities task 
followed by the truth task $(10 \%)$ and from three-valued to two-valued when they first received the truth task and then the possibilities task (8\%). This observed shift is meaningful since none of the participants shifts in the opposite direction, confirming the claim that next to individual differences, task characteristics also influence the results: For one fifth of the participants, task characteristics are dominant and cause them to shift in the predicted direction.

With respect to the relation between truth table classifications and cognitive ability, as in Experiment 1 a similar pattern of results was revealed for both measures of cognitive ability. Considering the possibilities task and the truth task together (see Table 4), there was a positive correlation between the CRT and both the FA-I and the ST-I. We also found an association between the Gospan and the FA-I and between the Gospan and the ST-I, but the latter failed to reach significance.

Insert Tables 4, 5 and 6 about here

Considering the possibilities task and the truth task separately (see respectively Table 5 and Table 6), a similar pattern of correlations was observed. For both tasks there was a positive association between the cognitive ability measures and both the FA-I and the ST-I. This correlational trend only reached significance with the CRT in the possibilities task and in the truth task only the correlation with the Gospan was significant.

As we discussed in the previous experiment, these positive associations between cognitive ability and the classification of false-antecedent cases as 'irrelevant' are evidence for the suppositional theory (Evans \& Over, 2004; Evans et al., 2007, 2008). In the present experiment, no significant correlations were revealed between the CRT or the Gospan and the MI-I. The mental models theory would predict participants with low cognitive ability to judge 
false-antecedent cases as 'irrelevant' and participants with high cognitive ability to judge the false-antecedent cases as 'possible' or 'true'. And this is not what is observed.

\section{Experiment 3}

As we discussed in Experiment 1 and Experiment 2, the positive associations between cognitive ability and the judgment of false-antecedent cases as 'irrelevant' are evidence for the suppositional theory (Evans \& Over, 2004; Evans et al., 2007, 2008), suggesting 'irrelevant' to be a meaningful option not only in the truth task but also in the possibilities task, with false antecedent cases yielding a truth value gap in a similar way as in the truth task (Evans \& Over, 2004; Sevenants et al., 2008).

One might argue however that the finding in Experiment 1 and 2 that 'irrelevant' is chosen as the judgment for the false antecedent cases considerably often in the possibilities task, might be due to the fact that people are confused by this option in a possibilities task. We do not believe this to be the case because of several reasons. Firstly, the participants making this irrelevance judgment in the possibilities task are the ones scoring the highest on the intelligence measures. It seems to us that if choosing irrelevant would be due to confusion or a misunderstanding of the task, it would be the ones with the least cognitive resources that would be the most susceptible to this, and not the ones with the highest intelligence score. Secondly, in previous experiments (Sevenants et al., 2008) considerably high rates of irrelevance judgments in the possibilities task were observed and justifications for these judgments were collected. None of the participants reported to have noticed anything strange about the task, or to have chosen this option because of confusion or as an alternative for "I don't know". Instead, almost all justifications were in terms of a truth value gap (see also Politzer, Over \& Baratgin, 2010; Evans \& Over, 2004). 
All parts of our experiments are presented in Dutch, and the denotation of 'irrelevant' in this language (niet ter zake doend, http://www.vandale.nl) is very close to the English meaning of this word: not useful or not relating to a particular situation, and therefore not important (Longman Modern English Dictionary, http://www.ldoceonline.com/dictionary/irrelevant), not related to what is being discussed or considered and therefore of no importance (Cambridge Advanced Learner's Dictionary, http://dictionary.cambridge.org/dictionary/british/irrelevant), having no bearing on or connection with the subject at issue (Webster Dictionary, http://www.websterdictionary.org/definition/irrelevant). This meaning, closely related to the truth value gap interpretation, is exactly the meaning participants are believed to ascribe to the option 'irrelevant' in the present truth table tasks, both in the possibilities and in the truth version. Consider the following statement from Evans and Over (2004, p. 40):

If we are in London then we have a good choice of theatres.

According to Ramsey Test, we can evaluate this conditional regardless of where we happen to be when it is asserted. No matter if we are in Durham or Plymouth (which have a poor choice of theatres) or in Paris (which has a great choice). What we do is to imagine that we are in London, making the least possible change to the actual world (not, for example, supposing that many London theatres have gone out of business). So perhaps the participants in these experiments are saying, in effect, it is irrelevant to the truth of the conditional that I am in Plymouth or Paris when the statement applies to London. We cannot move to the conclusion that they are saying that the truth of the conditional is indeterminate when the antecedent is false, which would contradict the Ramsey Test.

In the three-option possibilities task, participants are presented with the instruction "to evaluate for each of the four situations whether 'the situation is possible according to the rule', 'the situation is impossible according to the rule', or whether 'the situation is irrelevant according to the rule"'. We assume 'irrelevant' to be interpreted as 'not related to whatever is being considered, discussed' (Cambridge Advanced Learner's Dictionary, http://dictionary.cambridge.org/dictionary/british/irrelevant). So when participants, who are presented with conditional rules that are only about cards depicting a ' $\mathrm{T}$ ', are confronted with 
cards that do not contain a $\mathrm{T}$, they believe 'irrelevant' to be a better option than possible/impossible or true/false: It's not that a false antecedent case (i.e., 'not a $T$ ') is not possible/true or not impossible/false, it's just that it is not related to whatever is being considered (i.e., the conditional rule about 'a T').

Nevertheless, we are aware of the fact that there are reasons to be suspicious about 'irrelevant' as option in a truth table task, especially in the possibilities task. 'Irrelevant' has not exactly the same status in the possibilities task than it has in the truth task. In the truth task the three answer alternatives map onto distinctions made in three-valued logical systems that include indeterminate as a third option, in addition to 'true' and 'false'. Some situations make the rule true, other situations make it false and still some other situations do not provide any information about the truth of the conditional. So the three response options are exclusive. It might be harder to see how 'possible', 'impossible' and 'irrelevant' map onto exclusive response sets in the same way as in the truth task. Strictly speaking, the fact that a situation is possible according to the rule does not exclude it to be irrelevant according to that same rule. However, the option 'possible' in our possibilities task is conceived as credible or possible to conceive or imagine, so as a synonym of imaginable, conceivable (http://www.websterdictionary.org/definition/possible) and 'impossible' as incredible or totally unlikely and as a synonym of unimaginable or inconceivable (http://www.websterdictionary.org/definition/impossible). This is a meaning that also applies for the Dutch version of the task (mogelijk - zo dat het kan gebeuren; http://www.vandale.nl). Adding "irrelevant" as a third option to the possibilities task allows us to investigate whether there is space between "possible" and impossible", as is the case in the truth task with "irrelevant". "Irrelevant" in the possibilities task would then mean "doing nothing for credibility or imaginability". In that sense, adding irrelevant to the possibilities task does not make it that 
different from the situation in the truth task with irrelevant as a third option next to 'true' and 'false', where the three options map onto exclusive categories.

In the present experiment, we want to shed light on how people interpret the threeoption possibilities task and whether they have a clear understanding of it. So next to the justifications collected in Sevenants et al. (2008), it is aimed to clarify what the status of irrelevant is in the possibilities task and more specifically whether participants interpret this option in a similar way in both tasks. Participants are presented with exactly the same truth task or possibilities task as in the previous experiments of the present study, but additionally they are asked to rate each of the four situations on two scales: possible plus impossible for the possibilities task and true plus false for the truth task. Assuming that 'irrelevant' is interpreted in terms of a truth value gap (Evans \& Over, 2004), meaning 'not related to whatever is being considered, discussed' (Longman Modern English Dictionary), it is predicted that participants judging a situation to be 'irrelevant' will not give an extremely low rating of that situation on the possible/impossible or true/false scales (i.e., 0/1) but will instead rate this situation close to the midpoint (i.e., 4). Completing a Ramsey test (and thus considering both $\mathrm{A} \mathrm{C}$ and $\mathrm{A} \neg \mathrm{C}$ ), they are implying by their rating, accompanying an irrelevance judgment: It is not the case that this situation is not possible/impossible or true/false, it's just that this situation has nothing to do with the conditional rule and is therefore irrelevant with respect to the rule.

\section{Method}

\section{Participants}

Four hundred and eighty four first-year Psychology undergraduate students (17-23 years of age, $M=18.3$ ) at the University of Leuven participated on a voluntary basis. No participants had ever taken course in logic. The three-option possibilities task was completed by 230 
participants, while 254 participants completed the truth task. Students were randomly assigned to the task-type.

\section{Materials and Procedure}

Participants were run in two groups, with one group evaluating the four items of the threeoption possibilities task (TT, TF, FT and FF) and the other group evaluating the four items of the truth task as used in the previous experiments. Unlike Experiment 1 and 2, participants in the present experiment were presented only one run of the task and task-type therefore was a between-subjects variable. The tasks themselves were exactly the same as in the previous experiment, except that they were now presented in printed booklets, which participants completed in a self-paced manner. The instructions appeared on the first page of the booklet. For both task-types, participants were then successively presented with four pages in random order, containing both the conditional rule and one of the four combinations of the occurrence and non-occurrence of $\mathrm{A}$ and $\mathrm{C}$.

Each page of the booklet also contained two rating scales: Next to the evaluation of the possibility/truth of the four cases of the truth table task, participants in the present experiment were asked to rate their each of the four presented situation on two seven-point scales: For the possibilities task, they had to indicate on the first scale how possible the situation was according to the conditional rule with 1 being the least and 7 the most. On the second scale, they had to rate how impossible the situation was on a seven-point scale. Similarly, for every situation presented in the truth task, participants had to indicate on the first scale how true the situation was according to them, and on a second scale how false it was. No feedback or correct answers were provided. 


\section{Results and discussion}

Analyzing the data, we first examined participants' answer patterns (see Table 7), in order to check whether we could replicate the global picture of the patterns and the difference between the possibilities task and the truth task observed the previous experiments. Since participants only judged four situations (TT, TF, FT and FF) we examined the answer patterns and did not compute any indices as we did in the previous experiments. In the possibilities task, $50 \%$ of the participants responded according to a three-valued pattern (i.e., TFII, TFIT, TFIF, TFTI and TFFI), 44\% yielded a two-valued pattern (i.e., TFTT, TFFT, TFFF) and $6 \%$ of the answer patterns was categorized as 'other'. In the truth task, $75 \%$ of the response patterns was threevalued, $19 \%$ two-valued and $6 \%$ of the participants yielded an answer pattern that was categorized as "other". Overall, a Chi-square test revealed that on the one hand the proportion of two-valued answer patterns in the possibilities task was higher than the proportion of twovalued answer patterns in the truth task $\left(\chi^{2}(1)=39.15, p<.01\right)$. The proportion of threevalued patterns on the other hand was higher in the truth task than in the possibilities task $\left(\chi^{2}(1)=32.96, p<.01\right)$.

Insert Table 7 about here

Replicating the answer patterns of Sevenants et al. (2008) and those of Experiment 1 and 2, that more two-valued patterns are observed in the possibilities task than in the truth task, and that more three-valued patterns are observed with the truth task than with the possibilities-task, means that we can safely assume that the information we will derive from the rating scales also applies to other experiments in which the same tasks were used. The observation that only $6 \%$ of the judgments had to be categorized as 'other', both in the 
possibilities task and in the truth task suggests that both tasks were equally well understood, causing little confusion.

Regarding the rating scales, first of all the mean of the ratings for each scale separately per situation and per judgment was computed (see Appendix D), and this for both task-types. In a second step, the mean absolute value of the difference scores of the scales was calculated, again per situation and per value. A first observation (see Table 7) is that there was a significant difference in mean absolute value between the 'true' or 'false' judgments on the one hand and the 'irrelevant' judgments on the other hand. (This difference was not significant for the TF case, but this is caused by the fact that only 24 out of 484 participants did not give a false judgment for this case, see Appendix D). Ratings of 'possible/true' and 'impossible/false' are at the extremes of the scales when participants judge the situation to be 'possible/true' or 'impossible/false', meaning that a low rating on the first scale leads to a high rating on the second scale. 'Irrelevance' judgments however (almost solely observed with the false antecedent cases, see Table 6) are accompanied by a mean absolute value of the difference scores of the scales that is significantly lower than the mean difference scores accompanying 'possible/true' or 'impossible/false' judgments (see Table 7). More concrete, when a participants judges a case to be irrelevant, he tends to give a rating at the midpoint of both scales $(M=3.73$ for FT and 3.50 for FF in the possibilities task and 3.35 for FT and 3.57 for FF in the truth task, see respectively Table D1 and D2 of Appendix D).

A second observation is that regarding the ratings there is little difference between the possibilities task and the truth task (see Table 8). Regarding the mean of the absolute value of the difference scores accompanying the irrelevance judgments, for none of the cases a difference between the possibilities task and the truth task is observed. Taken together with the justifications collected by Sevenants et al. (2008) this suggests that 'irrelevant' is 
interpreted in a similar way in both tasks and thus can be considered a meaningful option in both tasks.

Insert Table 8 about here

Three differences are observed however between the possibilities task and the truth task regarding the difference scores, in which there seems to be a larger difference between the rating scales for the possibilities task than for the truth task. The first is for the TF case, where the absolute value of the difference score accompanying the impossible respective false judgments is higher for the possibilities task than for the truth task. Exactly the same goes for the FT case. This means that for the TF case and the FT case more extreme opposite ratings accompany the impossible judgments than false judgments. Finally, for the difference scores accompanying the possible respective true ratings on the FF case, more extreme opposite ratings are observed in the possibilities task than in the truth task. Overall, there seems to be a more pronounced distinction between the possible-scale and the impossible-scale in the possibilities task than there is between the true-scale and the false-scale in the truth task.

Globally, the administration of the rating scales revealed three elements. Firstly, there is a significant difference in the absolute value of the difference scores of the ratings scales accompanying an irrelevance judgment and accompanying a possible/true or impossible/false judgment. Irrelevance judgments are accompanied by two ratings at about the midpoint of the scales, whereas possible/true and impossible/false are accompanied by opposite ratings at the extremes of the two rating scales. Secondly, there seems to be no difference between the possibilities task and the truth task regarding the nature of the ratings accompanying irrelevance judgments. Finally, when differences between the possibilities task and the truth task are observed, it is the case that the possibilities task yields a larger absolute value of the 
difference scores of the ratings than does the truth task, meaning that in the possibilities task possible and impossible ratings are linked with more extreme opposite ratings on the possible and impossible scale than the true and false ratings are linked to in the truth task. These findings, taken together with the justifications collected by Sevenants et al. (2008), suggest that participants do not have difficulties interpreting the three-options possibilities task and do have a clear and coherent understanding of it not that differing from the interpretation of the three-option truth task.

We acknowledge however that 'irrelevant' does not have an unambiguous interpretation, not even in the truth task, and that there are other valuable third options instead of 'irrelevant', each of them having its own advantages and drawbacks. Politzer et al. (2010) make use of the term 'void' or 'neither true nor false', following the terminology of de Finetti (1937/1964; 2008) and Barrouillet et al. (for example, 2008) add 'one cannot know' to the options 'true' and 'false'. Schroyens (2010) even argues for a fourth response option which expresses that for general conditionals a case, though consistent, neither makes the rule false nor makes it irrefutably true. A wealth of experiments can be found in literature however in which the option 'irrelevant' is explicitly used in the standard truth table task (see for example Evans et al., 2007; Oberauer et al, 2007; Newstead, Ellis, Evans, \& Dennis, 1997). Enabling us to relate our findings to those in the literature is the reason why 'irrelevant' was used in present experiments, both for the truth task and the possibilities task.

\section{General Discussion}

The results of all three experiments in the present study replicate the finding that a larger proportion of two-valued answer patterns is observed in the possibilities task than in the truth task, and that a larger proportion of three-valued answer patterns is observed in the truth task than in the possibilities task (Barrouillet et al., 2008; Sevenants et al., 2008). This finding 
leads us to the conclusion that, regardless of participants' cognitive ability or individual differences in the interpretation of conditionals, task characteristics have an important influence on the classification of cases in the truth table task (see also Dugan \& Revlin, 1990; Thompson, 2000). Johnson Laird (2006) accounts for this difference between the possibilities task and the truth task as follows: People make a different kind of judgment when they are asked to evaluate the truth or falsity of conditional, as is the case in the truth task, than when they are asked, as in the possibilities task, which combination is possible given a conditional rule. To list the possibilities all they have to do is understand a conditional and think of what is possible, so they enumerate the complete, fully explicit models of the conditional. However, to evaluate whether a conditional is true or false, they have to compare it with a state of affairs that they see, but they also have to be able to understand the meta-linguistic meaning of true and false. They are more likely in this case to rely on mental models than on an enumeration of the possibilities. This makes the truth task harder than the possibilities task and explains why more three-valued answer patterns are observed in the former than in the latter type of truth table task.

The claim of Johnson Laird (2006) can not suffice however as an explanation for the difference between both task-types observed in the present study. Both the possibilities task and the truth task involve three-valued responses and in both tasks, the propensity to produce three-valued responses is related to mental capacity and intelligence, with smarter individuals more prone to adopt three-valued representations. These three-valued responses are more frequent in the truth task than in the possibilities task. This could lead to the conclusion that the possibilities task is more difficult than the truth task, which is in direct contrast with the explanation of Johnson Laird (2006) for the difference between both task-types. However, this conclusion is contradicted by the observation that all the developmental studies show that the truth task is more difficult than the possibilities task, even when the number of possible 
alternatives is the same in both tasks (see for example, Gauffroy \& Barrouillet, in press). In other words, at the moment, there is no clear answer to the question why there are more threevalued responses in the truth-task than in the possibilities task.

Despite the higher degree of irrelevance judgments of the false antecedent cases observed in the truth task than in the possibilities task, the answer patterns show that when they have the opportunity to do so, a considerable amount of participants judge false antecedent cases as irrelevant in the possibilities task. This is incompatible both with the original mental models theory (Johnson-Laird \& Byrne, 1991; 2002) and with the modified version of this theory by Barrouillet et al. (2008a). Both theories have an account for the irrelevance judgments of false antecedent cases in the truth task, but they predict these cases to be judged as possible in the classical possibilities task. Of course they have no explicit predictions about irrelevance judgments in the possibilities task since the classical version of this task does not allow for three-valued answer patterns. It seems reasonable to assume however that adding 'irrelevant' as a third option to this possibilities task should not affect the underlying reasoning processes judging the cases with fully explicit models in a possibilities task. This in our opinion leads both the original mental models theory and the modified version to make similar predictions regarding the false antecedent cases in the classical twooptions as well as the three-options version of the possibilities task: Participants would judge these cases as 'possible' and not as 'irrelevant'. The fact that we do observe irrelevance judgments in the possibilities task as well as in the truth task, provides evidence for a suppositional representation of conditionals and is problematic for the mental models approaches.

A second aim of this study was to examine whether participants produce the same response pattern repeatedly classifying the cases of the truth table task. Inspecting the individual answer patterns it is observed that both within and across tasks one third up to half 
of the participants is consistent giving either a two-valued either a three-valued answer pattern twice. Inspecting participants' consistency across tasks, one fifth of the participants is 'inconsistent' and shifts from two-valued to three-valued or from three-valued to two-valued. Remarkable however is that all these participants shift in a very 'consistent' way: When the possibilities task is followed by the truth task, they shift from two-valued to three-valued and when the truth task is presented first, followed by the possibilities task, they shift from a three-valued to a two-valued answer pattern. For none of the participants there is a shift the other way around (i.e., three-valued on the possibilities task and two-valued on the truth task). We conclude that for these participants task characteristics are dominant and they make a meaningful shift: These participants might not be that consistent, they are clearly not just giving a random answer.

Exploring the relation between participants' cognitive ability and their classification of the cases in the truth table task, we come to the following conclusion: Both in Experiment 1 and in Experiment 2 we find a positive correlation between the false-antecedent indices and participants' cognitive ability. This means that the participants scoring high on the CRT and on the Gospan working memory capacity measure classify the false-antecedent cases FT and FF more often as 'irrelevant' with respect to the conditional rule than the participants with less cognitive ability. A negative correlation in Experiment 1 (and no correlation in Experiment 2) is observed between the cognitive ability measures and the material implication index, in which the false-antecedent cases are judged as 'impossible' or 'false' with respect to the conditional. The combination of these findings is problematic for the mental models theory (Johnson-Laird, 2006; Johnson-Laird \& Byrne, 1991, 2002; JohnsonLaird et al., 1992): According to the mental models theory, the people with the highest working memory capacity are able to look beyond the initial model (A C) and to flesh out into fully explicit models, including the false-antecedent cases. This in turn leads to two-valued 
answer patterns. Participants with lower working memory capacity however do not process beyond the initial model and classify the truth table cases according to a three-valued pattern. These predictions are not in line with our findings.

From Experiment 1 and Experiment 2 we conclude that the observed correlations between the cognitive ability measures and the computed classification indices provide evidence for the three-valued truth table representation of the suppositional theory, according to which participants classifying the truth table cases according to three-valued patterns are those scoring the highest on cognitive ability (Evans et al., 2007, 2008). These are the participants who are able to complete both stages of the Ramsey test and thus to consider both A $\mathrm{C}$ and $\mathrm{A} \neg \mathrm{C}$, but who judge the false antecedent cases as 'irrelevant' with respect to the truth of the rule, since the antecedent is not present. This seems to be the case both for the truth task and for the possibilities task when participants are also able to give an irrelevance judgment in the latter.

In Experiment 3 it was examined whether participants have a clear understanding of the three option possibilities task, in order to rule out the suspicion that three-valued patterns observed in this task are due to participants' confusion caused by the addition of 'irrelevant' to the classical two-option truth table task. From the observation that the nature of the irrelevance judgments does not differ in the possibilities task and the truth task, we conclude that confusion is not the reason participants choose this option. A next step should be an experiment in which a probability assessment is entirely made. In real life, we are rarely certain of a p \& q case from the truth table. The indicative becomes void when we are. Generally, we get evidence but cannot be certain that "p \& q" holds, and we evaluate "if p then q" in that light. For both the mental models theory and the suppositional theory, $\mathrm{P}($ if $\mathrm{p}$ then $\mathrm{q}$ ) should increase when there is evidence for "p \& q", and decrease when there is evidence for "p \& not-q". The two theories differ however in the "not-p" cases or false antecedent cases. These are irrelevant in the suppositional account for an assessment of $\mathrm{P}($ if $\mathrm{p}$ then $\mathrm{q}$ ), but not in the mental models theory. 
In sum, in the present study it was aimed to replicate the difference between both types of truth table tasks investigating participants' classification of false antecedent cases. Furthermore it was examined how participants' answer patterns relate to their cognitive ability. Finally, it was explored whether participants have a clear understanding of the threeoption possibilities task. We replicate that more irrelevance classifications are yielded by the truth task than by the possibilities task, implying that task differences have an important influence on the results. Moreover, our data suggest that participants with high cognitive ability seem to be more susceptible to the appropriate task characteristics than participants with less cognitive ability. Secondly, we do observe however that up to half of the participants is consistent in giving either a two-valued or a three-valued classification of the truth table cases twice, both within and between tasks. This means that they classify the cases according to their individual interpretation of the conditional, regardless of the task characteristics. Moreover, there is a relation between participants' cognitive ability and their manner of classification: The higher participants are in cognitive ability, the more they respond according to a three-valued truth table, judging false antecedent cases to be irrelevant. Finally, it seems that these are genuine irrelevance judgments, not just caused by a misunderstanding of irrelevant in the possibilities task. The sum of these findings provides support for the suppositional theory. 


\section{References}

Adams, E. (1998). A Primer of Probability Logic. Stanford: CLSI publications.

Barrouillet, P., \& Lecas, J. F. (1998). How can mental models theory account for content effects in conditional reasoning? A developmental perspective. Cognition, 67, 209-253.

Barrouillet, P., Gauffroy, C., \& Lecas, J. F. (2008a). Mental models and the suppositional account of conditionals. Psychological Review, 115, 760-771.

de Finetti, B. (1937/1964). Foresight: Its logical laws, its subjective sources. Translated in H. E. Kyburg \& H. E. Smokier (eds), Studies in subjective probability (pp. 55-118). New York: Wiley.

de Finetti, B. (1967). Sur quelques conventions qui semblent utiles. [On some conventions that seem useful]. Revue Roumaine de Mathématiques Pures et Appliquées, xii, $1227-$ 1234.

de Finetti, B. (2008). Philosophical Lectures on Probability collected, edited, and annotated by Alberto Mura (Vol. 340). Berlin: Springer.

De Neys, W., d'Ydewalle, G., Schaeken, W., \& Vos, G. (2002). A Dutch, computerized, and group administrable adaptation of the operation span test. Psychologica Belgica, 42, 177-190.

Douven, I. \& Verbrugge, S. (2010). The Adams Family, Cognition, 117, 302-318.

Dugan, C. M., \& Revlin, R. (1990). Response options and presentation format as contributors to conditional reasoning. Quarterly Journal of Experimental Psychology, 42, 829-848.

Edgington, D. (2003). What if? Questions about conditionals. Mind and Language, 18, 380401.

Engle, R. W., Tuholski, S. W., Laughlin, J. E., \& Conway, A. R. A. (1999). Working memory, short-term memory, and general fluid intelligence: A latent-variable approach. Journal of Experimental Psychology: General, 128, 309-331. 
Evans, J. St. B. T., Over, D., (2004). If. Oxford University Press.

Evans, J. St. B. T., Over, D. E., \& Handley, S. H. (2003a). A theory of hypothetical thinking. In D. Hardman \& L. Maachi (Eds.), Thinking: Psychological perspectives on reasoning, judgement and decision making (pp. 3-22). Chichester: Wiley.

Evans, J. St. B. T., Handley, S. H., Neilens, H., \& Over, D. E. (2007). Thinking about conditionals: A study of individual differences. Memory \& Cognition, 35, 1722-1784.

Evans, J. St. B. T., Handley, S. H., Neilens, H., \& Over, D. E. (2008). Understanding causal conditionals: A study of individual differences. Quarterly Journal of Experimental Psychology, 61, 1291-1297.

Frederick, S. (2005). Cognitive reflection and decision making. Journal of Economic Perspectives, 19, 25-42.

\section{Gauffroy, C., \& Barrouillet, P. (in press). XXX, Journal of Developmental Psychology, x, xx-Xx.}

Heim, A. (1970). AH4 group test of general intelligence manual. London: Nelson.

Johnson-Laird, P. N. (2006). How we reason. New York: Oxford University Press.

Johnson-Laird, P. N., \& Byrne, R. M. J. (1991). Deduction. Hillsdale, NJ: Erlbaum.

Johnson-Laird, P. N., \& Byrne, R. M. J. (2002). Conditionals: A theory of meaning, pragmatics, and inference. Psychological Review, 109, 646-678.

Johnson-Laird, P. N., Byrne, R. M. J., \& Schaeken, W. (1992). Propositional reasoning by model. Psychological Review, 99, 418-439.

La Pointe, L. B., \& Engle, R. W. (1990). Simple and complex word spans as measures of working memory capacity. Journal of Experimental Psychology: Learning, Memory, and Cognition, 16, 1118-1133.

Navarrete, G., De Neys, W., \& Santamaria, C. (2008, July). Not so natural after all: Intelligence always matters. Paper presented at the XXIX International Congress of Psychology, Berlin, Germany. 
Newstead, S. E., Ellis, M. C., Evans, J. St. B. T., \& Dennis, I. (1997). Conditional reasoning with realistic material. Thinking and Reasoning, 3, 49-76.

Oberauer, K., Geiger, S., Fisher, K., \& Weidenfeld, A. (2007). Two meanings of 'if'? Individual differences in the interpretation of conditionals. Quarterly Journal of Experimental Psychology, 60, 790-819.

Oppenheimer, D. M. (2008). The secret life of fluency. Trends in Cognitive Science, 12, 237241.

Politzer, G., Over, D., \& Baratgin, J. (2010). Betting on conditionals. Thinking and Reasoning, 3, 172-197.

Ramsey, F. P. (1990). General propositions and causality (original publication, 1931). In D. H. Mellor (Ed.), Philosophical papers (pp. 145-163). Cambridge: Cambridge University Press.

Schroyens, W. (2010). A meta-analytic review of thinking about what is true, possible, and irrelevant in reasoning from or reasoning about conditional propositions. European Journal of Cognitive Psychology, 22, 897921.

Sevenants, A. Dieussaert, K., Schaeken, W. \& Barrouillet, P. (2009). Working memory and the truth table task. Unpublished manuscript.

Sevenants, A., Schroyens, W., Dieussaert, K., Schaeken, W., \& d'Ydewalle, G. (2008). Truth table tasks: The relevance of irrelevant. Thinking \& Reasoning, 14, 409-433.

Thompson, V. A. (2000). The task-specific nature of domain-general reasoning. Cognition, 76, 209-268.

Wason, P. C. (1966). Reasoning. In B. M. Foss (Ed.), New Horizons in Psychology I (pp. 10637). Harmandsworth: Penguin.

Wason, P. C., \& Johnson-Laird, P. N. (1969). Proving a disjunctive rule. Quarterly Journal of Experimental Psychology, 21, 14-20. 
Table 1

Correlation Matrix Showing the Cognitive Ability Measures and the Indexes of both Truth Table Tasks in Experiment $1(n=91)$

\begin{tabular}{|c|c|c|c|c|c|c|c|c|c|}
\hline & Mean & $\mathrm{Sd}$ & CRT & $\mathrm{GO}$ & Log-I & Conj-I & FA-I & ST-I & MI-I \\
\hline CRT & 1,75 & 1,08 & 1,00 & $0,23^{*}$ & 0,08 & $-0,28 *$ & $0,25 *$ & $0,26 *$ & $-0,02$ \\
\hline GO & 36,49 & 9,13 & & 1,00 & $-0,10$ & $-0,10$ & $0,25^{*}$ & $0,22 *$ & $-0,22^{*}$ \\
\hline Log-I & 3,89 & 0,31 & & & 1,00 & 0,11 & 0,05 & $0,26^{*}$ & $0,30 *$ \\
\hline Conj-I & 4,82 & 1,01 & & & & 1,00 & $-0,34 *$ & $-0,31^{*}$ & $-0,30^{*}$ \\
\hline FA-I & 1,68 & 1,42 & & & & & 1,00 & $0,98 *$ & $-0,70^{*}$ \\
\hline ST-I & 5,57 & 1,47 & & & & & & 1,00 & $-0,62 *$ \\
\hline MI-I & 5,27 & 1,49 & & & & & & & 1,00 \\
\hline
\end{tabular}


Table 2

Correlation Matrix Showing the Cognitive Ability Measures and the Indexes of the Possibilities Task in Experiment $1(n=47)$

\begin{tabular}{|c|c|c|c|c|c|c|c|c|c|}
\hline & Mean & $\mathrm{Sd}$ & CRT & GO & Log-I & Conj-I & FA-I & ST-I & MI-I \\
\hline CRT & 1,83 & 1,11 & 1,00 & 0,24 & 0,14 & $-0,29 *$ & 0,22 & 0,25 & 0,04 \\
\hline GO & 36,38 & 9,09 & & 1,00 & $-0,15$ & $-0,17$ & $0,51 *$ & $0,46^{*}$ & $-0,40 *$ \\
\hline Log-I & 3,89 & 0,31 & & & 1,00 & 0,23 & $-0,05$ & 0,20 & $0,34 *$ \\
\hline Conj-I & 4,55 & 0,85 & & & & 1,00 & $-0,18$ & $-0,12$ & $-0,34 *$ \\
\hline FA-I & 1,21 & 1,23 & & & & & 1,00 & $0,97 *$ & $-0,77 *$ \\
\hline ST-I & 5,11 & 1,26 & & & & & & 1,00 & $-0,67 *$ \\
\hline MI-I & 6,02 & 1,45 & & & & & & & 1,00 \\
\hline
\end{tabular}


Table 3

Correlation Matrix Showing the Cognitive Ability Measures and the Indices of the Truth Task in Experiment $1(n=44)$

\begin{tabular}{|c|c|c|c|c|c|c|c|c|c|}
\hline & Mean & $\mathrm{Sd}$ & CRT & $\mathrm{GO}$ & Log-I & Conj-I & FA-I & ST-I & MI-I \\
\hline CRT & 1,66 & 1,06 & 1,00 & 0,23 & 0,02 & $-0,25$ & $0,38 *$ & $0,36^{*}$ & $-0,24$ \\
\hline GO & 36,61 & 9,27 & & 1,00 & $-0,05$ & $-0,05$ & 0,04 & 0,03 & $-0,03$ \\
\hline Log-I & 3,89 & 0,32 & & & 1,00 & 0,04 & 0,15 & $0,35^{*}$ & $0,37 *$ \\
\hline Conj-I & 5,11 & 1,08 & & & & 1,00 & $-0,71 *$ & $-0,66^{*}$ & $-0,03$ \\
\hline FA-I & 2,18 & 1,45 & & & & & 1,00 & $0,98^{*}$ & $-0,55^{*}$ \\
\hline ST-I & 6,07 & 1,53 & & & & & & 1,00 & $-0,45^{*}$ \\
\hline MI-I & 4,48 & 1,07 & & & & & & & 1,00 \\
\hline
\end{tabular}


Table 4

Correlation Matrix Showing the Cognitive Ability Measures and the Indexes of both Truth Table Tasks in Experiment $2(n=77)$

\begin{tabular}{|c|c|c|c|c|c|c|c|c|c|}
\hline & Mean & $\mathrm{Sd}$ & CRT & $\mathrm{GO}$ & Log-I & Conj-I & FA-I & ST-I & MI-I \\
\hline CRT & 1,38 & 1,12 & 1,00 & $0,39 *$ & $0,34 *$ & 0,02 & $0,30 *$ & $0,42 *$ & 0,16 \\
\hline GO & 35,42 & 8,83 & & 1,00 & 0,04 & $-0,05$ & $0,23 *$ & 0,20 & $-0,11$ \\
\hline Log-I & 3,65 & 0,81 & & & 1,00 & $0,67^{*}$ & 0,11 & $0,63^{*}$ & $0,52^{*}$ \\
\hline Conj-I & 5,13 & 1,40 & & & & 1,00 & $-0,41 *$ & 0,05 & 0,15 \\
\hline FA-I & 1,51 & 1,14 & & & & & 1,00 & $0,84^{*}$ & $-0,37 *$ \\
\hline ST-I & 5,16 & 1,47 & & & & & & 1,00 & 0,00 \\
\hline MI-I & 4,66 & 1,06 & & & & & & & 1,00 \\
\hline
\end{tabular}


Table 5

Correlation Matrix Showing the Cognitive Ability Measures and the Indexes of the Possibilities Task in Experiment 2 (n=77)

\begin{tabular}{|c|c|c|c|c|c|c|c|c|c|}
\hline & Mean & $\mathrm{Sd}$ & CRT & $\mathrm{GO}$ & Log-I & Conj-I & FA-I & ST-I & MI-I \\
\hline CRT & 1,38 & 1,12 & 1,00 & $0,39 *$ & $0,30 *$ & 0,04 & 0,15 & $0,30^{*}$ & 0,17 \\
\hline GO & 35,42 & 8,83 & & 1,00 & 0,03 & $-0,05$ & 0,11 & 0,11 & $-0,02$ \\
\hline Log-I & 1,81 & 0,46 & & & 1,00 & $0,69 *$ & $-0,09$ & $0,51^{*}$ & $0,51 *$ \\
\hline Conj-I & 2,55 & 0,84 & & & & 1,00 & $-0,38 *$ & 0,08 & 0,07 \\
\hline FA-I & 0,60 & 0,67 & & & & & 1,00 & $0,81 *$ & $-0,56^{*}$ \\
\hline ST-I & 2,40 & 0,78 & & & & & & 1,00 & $-0,18$ \\
\hline MI-I & 2,47 & 0,79 & & & & & & & 1,00 \\
\hline
\end{tabular}


Table 6

Correlation Matrix Showing the Cognitive Ability Measures and the Indexes of the Truth Task in Experiment $2(n=77)$

\begin{tabular}{|c|c|c|c|c|c|c|c|c|c|}
\hline & Mean & $\mathrm{Sd}$ & CRT & GO & Log-I & Conj-I & FA-I & ST-I & MI-I \\
\hline CRT & 1,38 & 1,12 & 1,00 & $0,39 *$ & $0,31 *$ & $-0,01$ & $0,30 *$ & $0,41 *$ & 0,06 \\
\hline GO & 35,42 & 8,83 & & 1,00 & 0,05 & $-0,05$ & $0,24 *$ & $0,23 *$ & $-0,18$ \\
\hline Log-I & 1,84 & 0,43 & & & 1,00 & $0,65^{*}$ & 0,03 & $0,50 *$ & $0,47 *$ \\
\hline Conj-I & 2,58 & 0,85 & & & & 1,00 & $-0,54^{*}$ & $-0,17$ & $0,24 *$ \\
\hline FA-I & 0,91 & 0,80 & & & & & 1,00 & 0,88 & $-0,51 *$ \\
\hline ST-I & 2,75 & 0,92 & & & & & & 1,00 & $-0,22$ \\
\hline MI-I & 2,19 & 0,61 & & & & & & & 1,00 \\
\hline
\end{tabular}


Table 7

T-Tests per Case of the Absolute Value of the Difference Scores in Experiment 3( $n=484)$

\begin{tabular}{|c|c|c|c|c|c|c|c|c|}
\hline Case & Option & Mean X & Mean Y & SD X & SD Y & t-value & $\mathrm{p}$-value & $\mathrm{df}$ \\
\hline & 1 vs 0 & 2.25 & 5.54 & 2.63 & 1.24 & -5.24 & $<.01$ & 462 \\
\hline \multirow[t]{3}{*}{$\mathrm{TF}$} & 2 vs 0 & 1.80 & 5.54 & 2.21 & 1.24 & 12.71 & $<.01$ & 478 \\
\hline & 1 vs 2 & 2.25 & 1.80 & 2.63 & 2.21 & 0.72 & .72 & 22 \\
\hline & 1 vs 0 & 3.69 & 5.06 & 2.03 & 1.81 & -5.40 & $<.01$ & 303 \\
\hline \multirow[t]{3}{*}{ FT } & 2 vs 0 & 1.60 & 5.06 & 2.02 & 1.81 & -18.27 & $<.01$ & 412 \\
\hline & 1 vs 2 & 3.69 & 1.60 & 2.03 & 2.02 & 7.29 & $<.01$ & 247 \\
\hline & 1 vs 0 & 5.16 & 5.38 & 1.51 & 1.33 & -0.83 & 0.36 & 218 \\
\hline \multirow[t]{2}{*}{ FF } & 2 vs 0 & 1.36 & 5.38 & 2.05 & 1.33 & -11.99 & $<.01$ & 302 \\
\hline & 1 vs 2 & 5.16 & 1.36 & 1.51 & 2.05 & 21.24 & $<.01$ & 442 \\
\hline
\end{tabular}

Note - 1 stands for 'true' in the truth task and for 'possible' in the possibilities task; 0 stands for 'false' in the truth task and for 'impossible' in the possibilities task; 2 stands for 'irrelevant', both in the truth task and the possibilities task 
Table 8

T-Tests per Task of the Absolute Value of the Difference Scores in Experiment 3(n PTT = $230 ; N T T T=254)$

\begin{tabular}{|c|c|c|c|c|c|c|c|c|}
\hline Case & & $\begin{array}{c}\text { Mean } \\
\text { PTT }\end{array}$ & $\begin{array}{c}\text { Mean } \\
\text { TTT }\end{array}$ & $\begin{array}{c}\text { SD } \\
\text { PTT }\end{array}$ & $\begin{array}{c}\text { SD } \\
\text { TTT }\end{array}$ & t-value & p-value & df \\
\hline \multirow[t]{2}{*}{ TT } & 0 & 5.25 & 5.59 & 1.13 & 1.18 & 0.21 & 0.83 & 482 \\
\hline & 0 & 5.70 & 5.40 & 0.97 & 1.42 & 2.73 & $<.01$ & 458 \\
\hline \multirow{2}{*}{$\mathrm{TF}$} & 2 & 0.60 & 2.20 & 0.55 & 2.42 & -1.44 & 0.17 & 18 \\
\hline & 0 & 5.51 & 4.70 & 1.24 & 2.09 & 3.52 & $<.01$ & 233 \\
\hline \multirow[t]{3}{*}{ FT } & 1 & 3.66 & 4.00 & 2.09 & 1.22 & -0.36 & 0.72 & 68 \\
\hline & 2 & 1.35 & 1.74 & 1.83 & 2.11 & -1.20 & 0.23 & 177 \\
\hline & 0 & 5.63 & 5.21 & 0.81 & 1.59 & 0.97 & 0.34 & 38 \\
\hline \multirow[t]{2}{*}{$\mathrm{FF}$} & 1 & 5.36 & 4.80 & 1.25 & 1.85 & 2.44 & $<.05$ & 178 \\
\hline & 2 & 1.16 & 1.48 & 1.81 & 2.18 & -1.20 & 0.23 & 262 \\
\hline
\end{tabular}




\section{APPENDIX A}

\section{Table A1}

Response Patterns (\%) for the Possibilities Tasks and the Truth Tasks in Experiment 1

\begin{tabular}{ccc}
\hline TFTT & $\begin{array}{c}\text { Possibilities Task } \\
(n=47)\end{array}$ & $\begin{array}{c}\text { Truth Task } \\
(n=44)\end{array}$ \\
TFFT & 17 & 5 \\
TFII & 10 & 14 \\
TFFI & 11 & 32 \\
TFFF & 0 & 32 \\
TFIT & 16 & 5 \\
TFTI & 6 & 5 \\
Other & 9 & 0 \\
\hline
\end{tabular}

\section{Table A2}

Grouping (\%) of Response Patterns of Experiment 1 into Two-Valued and Three-Valued Patterns

\begin{tabular}{cccc}
\hline $\begin{array}{c}\text { Possibilities Task - } \\
\text { Possibilities Task }\end{array}$ & $\begin{array}{c}\text { Truth Task - } \\
\text { Truth Task }\end{array}$ & Total \\
\hline $2-2$ & 36 & 9 & 23 \\
$3-3$ & 13 & 56 & 33 \\
$2-3$ & 4 & 7 & 5 \\
$3-2$ & 2 & 5 & 3 \\
Other & 45 & 23 & 36 \\
\hline Total & 100 & 100 & 100 \\
\hline
\end{tabular}




\section{APPENDIX B}

\section{Table B1}

Response Patterns (\%) for the Possibilities Task and the Truth Task in Experiment 2

\begin{tabular}{ccc}
\hline TFTT & $\begin{array}{c}\text { Possibilities Task } \\
(n=77)\end{array}$ & $\begin{array}{c}\text { Truth Task } \\
(n=77)\end{array}$ \\
TFFT & 10 & 3 \\
TFII & 8 & 18 \\
TFFI & 19 & 23 \\
TFFF & 8 & 29 \\
TFIT & 5 & 10 \\
TFTI & 3 & 4 \\
Other & 21 & 0 \\
\hline
\end{tabular}

\section{Table B2}

Grouping (\%) of Response Patterns of Experiment 2 into Two-Valued and Three-Valued Patterns

\begin{tabular}{cccc}
\hline & $\begin{array}{c}\text { Possibilities Task - } \\
\text { Truth Task }\end{array}$ & $\begin{array}{c}\text { Truth Task - } \\
\text { Possibilities Task }\end{array}$ & Total \\
\hline $2-2$ & 13 & 18 & 16 \\
$3-3$ & 15 & 26 & 21 \\
$2-3$ & 21 & 0 & 10 \\
$3-2$ & 0 & 16 & 8 \\
Other & 51 & 39 & 45 \\
\hline Total & 100 & 100 & 100 \\
\hline
\end{tabular}




\section{APPENDIX C}

\section{Table C}

Response Patterns (\%) for the Possibilities Task and the Truth Task in Experiment 3

\begin{tabular}{ccc}
\hline TFTT & $\begin{array}{c}\text { Possibilities Task } \\
(n=230)\end{array}$ & $\begin{array}{c}\text { Truth Task } \\
(n=254)\end{array}$ \\
TFFT & 17 & 1 \\
TFII & 23 & 14 \\
TFFI & 17 & 30 \\
TFFF & 4 & 32 \\
TFIT & 8 & 4 \\
TFTI & 8 & 9 \\
Other & 6 & 9 \\
\hline
\end{tabular}




\section{APPENDIX D}

\section{Table D1}

Means of the Possible/Impossible Ratings for the Possibilities Task per Case in Experiment 3 $(n=230)$

\begin{tabular}{|c|c|c|c|c|c|c|}
\hline Case & $\mathrm{N}$ & Scale & Mean & $\mathrm{SD}$ & Minimum & Maximum \\
\hline \multirow{2}{*}{ TT 1} & \multirow{2}{*}{230} & Possible & 6.66 & 1.09 & 1 & 7 \\
\hline & & Impossible & 1.41 & 1.28 & 1 & 7 \\
\hline \multirow{2}{*}{ TF 0} & \multirow{2}{*}{221} & Possible & 1.19 & 0.68 & 1 & 6 \\
\hline & & Impossible & 6.82 & 0.66 & 2 & 7 \\
\hline \multirow{2}{*}{ TF 1} & \multirow{2}{*}{4} & Possible & 3.25 & 1.50 & 1 & 4 \\
\hline & & Impossible & 4.00 & 2.16 & 2 & 7 \\
\hline \multirow{2}{*}{ TF 2} & \multirow{2}{*}{5} & Possible & 3.45 & 1.36 & 1 & 6 \\
\hline & & Impossible & 4.65 & 1.39 & 2 & 7 \\
\hline \multirow{2}{*}{ FT 0} & \multirow{2}{*}{103} & Possible & 1.40 & 1.12 & 1 & 7 \\
\hline & & Impossible & 6.66 & 1.1 & 1 & 7 \\
\hline \multirow{2}{*}{ FT 1} & \multirow{2}{*}{65} & Possible & 5.65 & 1.39 & 1 & 7 \\
\hline & & Impossible & 2.45 & 1.62 & 1 & 7 \\
\hline \multirow{2}{*}{ FT 2} & \multirow{2}{*}{62} & Possible & 4.37 & 1.23 & 1 & 7 \\
\hline & & Impossible & 3.73 & 1.42 & 1 & 7 \\
\hline \multirow{2}{*}{ FF 0} & \multirow{2}{*}{16} & Possible & 1.5 & 0.60 & 1 & 7 \\
\hline & & Impossible & 6.38 & 0.82 & 1 & 7 \\
\hline \multirow{2}{*}{ FF 1} & \multirow{2}{*}{116} & Possible & 6.67 & 0.60 & 4 & 7 \\
\hline & & Impossible & 1.31 & 0.82 & 0 & 7 \\
\hline \multirow{2}{*}{ FF 2} & \multirow{2}{*}{98} & Possible & 4.06 & 1.64 & 1 & 7 \\
\hline & & Impossible & 3.50 & 1.72 & 1 & 7 \\
\hline
\end{tabular}


Table D2

Means of the True/False Ratings per Case for the Truth Task in Experiment $3(n=254)$

\begin{tabular}{|c|c|c|c|c|c|c|}
\hline & $\mathrm{N}$ & Scale & Mean & $\mathrm{SD}$ & Minimum & Maximum \\
\hline \multirow{2}{*}{ TT 1} & \multirow{2}{*}{254} & True & 6.81 & 0.65 & 1 & 7 \\
\hline & & False & 1.31 & 1.08 & 1 & 7 \\
\hline \multirow{2}{*}{ TF 0} & \multirow{2}{*}{239} & True & 1.28 & 0.77 & 1 & 7 \\
\hline & & False & 6.67 & 0.76 & 3 & 7 \\
\hline \multirow{2}{*}{ TF 2} & \multirow{2}{*}{15} & True & 3.20 & 1.42 & 1 & 6 \\
\hline & & False & 4.73 & 1.53 & 2 & 7 \\
\hline \multirow{2}{*}{ FT 0} & \multirow{2}{*}{132} & True & 1.85 & 1.50 & 1 & 7 \\
\hline & & False & 6.02 & 1.63 & 1 & 2 \\
\hline \multirow{2}{*}{ FT 1} & \multirow{2}{*}{5} & True & 5.80 & 0.84 & 5 & 7 \\
\hline & & False & 1.80 & 0.45 & 1 & 7 \\
\hline \multirow{2}{*}{ FT 2} & \multirow{2}{*}{117} & True & .19 & 1.32 & 1 & 7 \\
\hline & & False & 3.35 & 1.44 & 1 & 7 \\
\hline \multirow{2}{*}{ FF 0} & \multirow{2}{*}{24} & True & 1.79 & 1.72 & 1 & 7 \\
\hline & & False & 6.50 & 1.29 & 1 & 7 \\
\hline \multirow{2}{*}{ FF 1} & \multirow{2}{*}{64} & True & 6.34 & 0.10 & 2 & 7 \\
\hline & & False & 1.55 & 1.01 & 1 & 5 \\
\hline \multirow{2}{*}{ FF 2} & \multirow{2}{*}{166} & True & 3.96 & 1.50 & 1 & 7 \\
\hline & & False & 3.57 & 1.15 & 1 & 7 \\
\hline
\end{tabular}

